\title{
Diagnóstico temprano del cáncer de próstata: Información para pacientes
}

\author{
Agustín Ciapponi๋, Carlos García ${ }^{\ddagger}$, Laura Fraguas ${ }^{\S}$, Gabriel Villalón ${ }^{\ddagger}$
}

Ciapponi A, García C, Fraguas L, Villalón G y Servicio de Medicina Familiar y Comunitaria del Hospital Italiano de Buenos Aires. Diagnóstico precoz del cáncer de próstata: Información para pacientes. Evid Act Pract Ambul. Abr-Jun 2012;15(2):64-65.

Está muy difundida la idea de que los hombres a partir de cierta edad deben hacerse exámenes destinados al diagnóstico temprano del cáncer de próstata (CP). Estos métodos incluyen el tacto rectal, una determinación sanguínea que se llama antígeno prostático específico (PSA, por su sigla en inglés) y/o la ecografía prostática.

La próstata es una glándula que se encuentra solo en los hombres y se ubica debajo de la vejiga y adelante del recto. Se dispone formando un anillo alrededor de la uretra, el conducto encargado de transportar la orina desde la vejiga hacia el exterior del cuerpo. Esta glándula participa en la formación del semen y puede ser objeto de algunas enfermedades, entre las más frecuentes: inflamación (prostatitis), crecimiento benigno (hiperplasia prostática benigna), y cáncer de próstata.

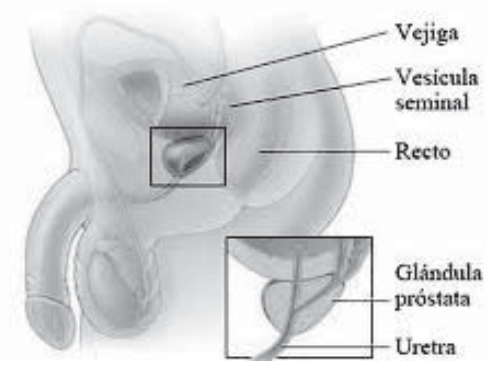

EI PSA es una proteína producida por la próstata. Los niveles de esta proteína aumentan en la sangre cuando un hombre tiene cáncer de próstata, pero también aumentan en las otras enfermedades benignas de la próstata que ya mencionamos, así como también en algunas situaciones fisiológicas, como al haber tenido relaciones sexuales o luego de andar en bicicleta (por compresión local transitoria).

A pesar del entusiasmo incial que han tenido algunos de los métodos ya mencionados (particularmente el PSA) en la prevención del $\mathrm{CP}$, también han ido apareciendo en forma creciente estudios científicos que cuestionan su utilidad. Particularmente, en mayo de 2012 la fuerza de tareas estadounidense de prácticas preventivas (USPSTF, por su sigla en inglés), una de las organizaciones con mayor peso a nivel mundial en lo que respecta a cuidados preventivos de la salud, finalmente se expidió en contra del diagnóstico temprano de CP utilizando al PSA en hombres de la población general, independientemente de su edad.

Tras un extenso y profundo debate, este organismo llegó a la conclusión de que hasta que se desarrollen nuevas técnicas preventivas muchos hombres se ven perjudicados como consecuencia del rastreo del CP y pocos, o ninguno, beneficiados.

Se describen aquí estimaciones de posibles beneficios y daños de los hombres sometidos a una prueba de PSA realizada cada uno a cuatro años durante un lapso de diez años:
Posible beneficio de la detección temprana del cáncer de próstata

Insistimos en que la reducción en las muertes por CP mediante el PSA es a lo sumo pequeña. Para ejemplificarlo con números podemos mencionar que, de acuerdo a datos de estudios que evaluaron cerca de 400.000 hombres, el realizar la determinación rutinaria de PSA reduce como máximo una muerte por $\mathrm{CP}$ por cada 1.000 hombres evaluados (por cada 1.000 pacientes que eligieron no hacerse el PSA durante diez años murieron cinco pacientes a consecuencia de un $\mathrm{CP}$, comparado con cuatro a cinco pacientes que murieron de CP aun habiendo realizado el PSA con cierta periodicidad).

\section{Daños esperados de la detección}

La mayoría de los cánceres de próstata que se encuentran como consecuencia de haber solicitado un PSA son de crecimiento lento, no amenazan la vida, y no causarán daños durante toda la vida de un hombre. Sin embargo, actualmente no hay manera de determinar qué tipos de cáncer es probable que amenacen la salud de un hombre y cuáles no. Por ende, casi todos los hombres con CP detectados por el PSA optan por recibir tratamiento. La prueba de detección del PSA a menudo sugiere que el CP puede estar presente aun cuando NO hay cáncer (esto en medicina se conoce como un resultado "falso positivo"). De hecho si todos los hombres "nos sacáramos la próstata", como se demostró en estudios que analizaron la próstata de hombres que habían muerto por otras causas (por ejemplo del corazón o accidentes), se detectaría un "cáncer de próstata" (en realidad, presencia de células tumorales que nunca llegan a afectar la salud de las personas) en 10 de cada 100 personas de entre 20 y 29 años; 30 de cada 100 entre los 30 y 39 años; 40 de cada 100 entre los 40 y 49 años; 45 de cada 100 entre los 40 y 59 años; 65 de cada 100 entre los 60 y 69 años; y 80 de cada 100 entre los 70 y 79 años. Estos resultados generan preocupación y ansiedad y pueden desencadenar pruebas de seguimiento y procedimientos, tales como biopsias innecesarias. Dado que las biopsias son punciones que atraviesan otros tejidos, pueden causar en un 33\% de las ocasiones daños tales como fiebre, infección, hemorragia, problemas urinarios y dolor. Incluso un $1 \%$ de hombres tiene que ser hospitalizado a causa de estas complicaciones. Una vez realizado el diagnóstico de $\mathrm{CP}$, las estrategias de tratamiento del CP localizado son: conducta expectante, vigilancia activa (dos variantes de esperar y seguir estudiando), cirugía y/o radioterapia. Además de las complicaciones asociadas al diagnóstico temprano y a la cascada diagnóstica que este puede generar, los daños secundarios al tratamiento del cáncer de próstata detectados por rastreo son claros y pueden ser graves.

Por cada 1.000 hombres que eligen realizar PSA:

- 100 a 120 tienen al menos un resultado falso positivo.

- Diez hombres se internan a causa de complicaciones de la biopsia prostática.

- Dos hombres experimentan un evento cardiovascular grave, como infarto de miocardio, debido al tratamiento del CP.

- Un hombre desarrolla trombosis venosa grave en los miembros inferiores o los pulmones debido al tratamiento. 
- 29 hombres desarrollan problemas de erección debido al tratamiento.

- 18 hombres desarrollan incontinencia urinaria (dificultad para retener la orina) debido al tratamiento.

Además, por cada 3.000 hombres que eligen realizarse el PSA: - Un hombre muere debido a complicaciones del tratamiento quirúrgico.

Es importante entender que muchos de estos hombres en realidad no necesitaban tratamiento, ya que su cáncer no habría crecido ni habría causado problemas de salud sin tratamiento.

Entonces.... ¿Cómo debo decidir si hacerme o no un PSA? Ud. debe saber la información actual sobre la detección de CP utilizando el PSA: existe un pequeño posible potencial y existen importantes daños. Pero también debe pensar acerca de sus propias creencias y preferencias personales para el cuidado de su salud, sopesar los beneficios y daños de la prueba de PSA, y decidir qué es más importante para Ud. Si existe la posibilidad, por pequeña que sea, de evitar la muerte por CP quizás sea esto más importante para Ud. que el riesgo a los daños ya mencionados y, en ese caso, hacerse el PSA puede ser la decisión correcta. Si por el contrario considera que estos daños son demasiado importantes como para justificar un beneficio no confirmado, no hacerse el PSA puede ser la decisión correcta. Su médico puede ayudarlo a entender el significado de la evidencia científica y a tomar una decisión que refleje sus valores y preferencias.

\section{Bibliografía}

1. USPSTF. Screening for Prostate Cancer. Current Recommendation (en línea). Disponible en URL: http://www.uspreventiveservicestaskforce.org/prostatecancerscreening.htm. Último acceso 20/6/2012

2. Ciapponi A. Adiós al rastreo de cáncer de próstata con las técnicas actuales. Evid Act Pract Ambul. Abr-Jun 2012;15(2):47 